\title{
Eosinophilic granulomatosis with polyangiitis in a 10-year-old girl
}

\author{
Huang Linxue MD, Mao Yujie MD PhD
}

- Cite as: CMAJ 2019 August 19;191:E919. doi: 10.1503/cmaj.190247

A 10-year-old girl with a history of asthma presented to our clinic with a 10-day history of painful skin lesions on her legs and difficulty walking. Physical examination showed bruises with necrosis on her left leg and cyanosis on her right foot, with decreased skin temperature (Figure 1). Laboratory tests showed an elevated eosinophil count of 7.33 (reference range $0.02-0.52$ ) $\times 10^{\%} / \mathrm{L}$, and an increased immunoglobulin $\mathrm{E}$ (IgE) level of 1710 (reference range 0-200) IU/mL. Antineutrophil cytoplasm antibody, parasite antibodies and a bone marrow test were negative. A computed tomography scan of the patient's chest showed 5-mm ground glass opacities (Appendix 1, available at www.cmaj.ca/lookup/ suppl/doi:10.1503/cmaj.190247/-/DC1). Ultrasound showed bilateral leg artery occlusions. Electromyography suggested severe damage to both motor and sensory fibres. Skin biopsy showed non-necrotizing vasculitis and minimal eosinophil infiltration of adjacent tissues. We diagnosed eosinophilic granulomatosis with polyangiitis according to the patient's history of asthma, peripheral hypereosinophilia, neuropathy, pulmonary infiltrates and extravascular eosinophil, meeting 5 of 6 of the 1990 American College of Rheumatology criteria for the condition. ${ }^{1}$ We prescribed prednisone at $1 \mathrm{mg} / \mathrm{kg}$ daily for 2 weeks, followed by gradual tapering. The patient's symptoms improved, but did not entirely resolve, over the next 2 months.

Eosinophilic granulomatosis with polyangiitis (formerly Churg-Strauss syndrome) is an uncommon systemic necrotizing vasculitis of small- and medium-size vessels that develops primarily in middle-aged individuals. It is characterized by asthma, blood eosinophilia and extrapulmonary manifestations. The condition is rarely described in childhood. Pulmonary and cardiac involvement is predominant in pediatric eosinophilic granulomatosis with polyangiitis, and mortality is substantial. ${ }^{2}$

Skin manifestations of the condition include purpura, nodules, livedo reticularis, Raynaud phenomenon and gangrenous necrotic lesions. Histologic characteristics include necrotizing vasculitis, granulomas and extravascular eosinophils. Because the simultaneous presence of all 3 types of pathologic features is uncommon, clinical history is important in making this diagnosis. ${ }^{3}$ Cutaneous lesions may be the initial manifestation, requiring extensive investigations to confirm the diagnosis. Delayed diagnosis and severe organ involvement affect the prognosis. ${ }^{2}$ The mainstay of treatment is glucocorticoids; immunosuppressants are indicated for patients with poor prognostic factors such as gastrointestinal, cardiac or renal involvement, or in people older than 65 years. ${ }^{3}$

\section{References}

1. Masi AT, Hunder GG, Lie JT, et al. The American College of Rheumatology 1990 criteria for the classification of Churg-Strauss syndrome (allergic granulomatosis and angiitis). Arthritis Rheum 1990;33:1094-100.

2. Zwerina J, Eger G, Englbrecht M, et al. Churg-Strauss syndrome in childhood: a systematic literature review and clinical comparison with adult patients. Semin Arthritis Rheum 2009;39:108-15.

3. Nguyen Y, Guillevin L. Eosinophilic granulomatosis with polyangiitis (ChurgStrauss). Semin Respir Crit Care Med 2018;39:471-81.

\section{Competing interests: None declared.}

This article has been peer reviewed.

The authors have obtained consent from the patient's parents.

Affiliation: Department of Dermatology and Venereology, Sichuan Academy of Medical Sciences and Sichuan Provincial People's Hospital, Chengdu, China.

Correspondence to: Mao Yujie, Yujiemao@yeah.net 